\title{
MSR I47WD: New wireless data logger for measuring the physiological properties for textiles
}

\section{News}

Seuzach, Switzerland, January 22, 2018 - Functional clothing is subjected to extensive testing, both in the laboratory and in the field, before entering the market. With the new MSR147WD wireless data logger, MSR Electronics wants to make the complex measuring and monitoring of physiological parameters such as skin temperature and moisture level easier, and make it accessible to a wide user field.

The Swiss technology company, which specializes in miniaturized data loggers and is based in Seuzach, has made a name for itself over the past ten years, primarily in the field of universal data loggers. The MSR loggers are primarily used in transportation and machine monitoring; occasionally they even accompany supply flights to the International Space Station (ISS) in order to provide the US space agency with detailed data on transportation stresses.

With the new MSR147WD wireless data logger, MSR Electronics $\mathrm{GmbH}$ is now expanding its series of "special data loggers". These include a range of type variants, from rumination sensors for researching animal behaviour, through to custom products for measuring voltage signals in rockfall protection systems. MSR's special data loggers are always developed in close co-operation with the customer; this also applies to the new MSR147WD, which has been designed for measuring the physiological properties of textiles and is already being successfully used in the textile and clothing industry.

\section{Plug-in sensors for greater flexibility}

The plug-in sensors for the MSR147WD supplied by MSR Electronics $\mathrm{GmbH}$ can be inserted and removed individually. Sensors measuring air pressure and 3-axis acceleration (static) are available as internal sensors; in addition, there are five connections for plug-in external, pre-calibrated humidity and temperature sensors. Due to the automatic configuration feature, the data logger instantly recognises when a sensor is inserted and which type.

\section{BLE for wireless monitoring}

The MSR147WD features Bluetooth Low Energy short-range radio technology (Bluetooth 4.0 Smart). "When testing functional clothing by measuring comfort on the skin and in the microclimate between the individual layers of clothing, BLE makes the work significantly easier.

Thanks to the wireless transfer of the measured data, the MSR smartphone app can be used to check whether the data is being properly recorded at any given point, without the test subject having to waste time to put on and take off the clothing," explains MSR CEO Wendelin Egli. Thanks to the integrated OLED screen in the data logger, the current measured data can be checked even while measuring takes place, without having to use a mobile phone. By means of the "MSR Data Logger" app, data recordings can be started, stopped, read out
Volume 4 Issue 2 - 2018

Gabriela Zumkehr

MSR Electronics GmbH, Switzerland

Correspondence: Gabriela Zumkehr, MSR Electronics GmbH, Mettlenstrasse 6, CH-8472, Seuzach, Switzerland, Tel +41523162555, Fax+41523163521, Email g.zumkehr@msr.ch

Received: January 22, 2018 | Published: March 02, 2018

and sent to the MSR Smart Cloud. MSR Smart Cloud facilitates the storage of measured data on a server via the Internet, allowing the user to view the measured data of the data logger wherever they are and to grant further participants access to his/her measured data, if required. The data can be read out of the MSR147WD via BLE and USB. The PC software for configuring the data logger and analysing the values is supplied free of charge by the manufacturer. Should the customer require advice when analysing the metrological data, MSR Electronics will provide an established specialist for measuring the physiological properties of textiles (Figure 1).

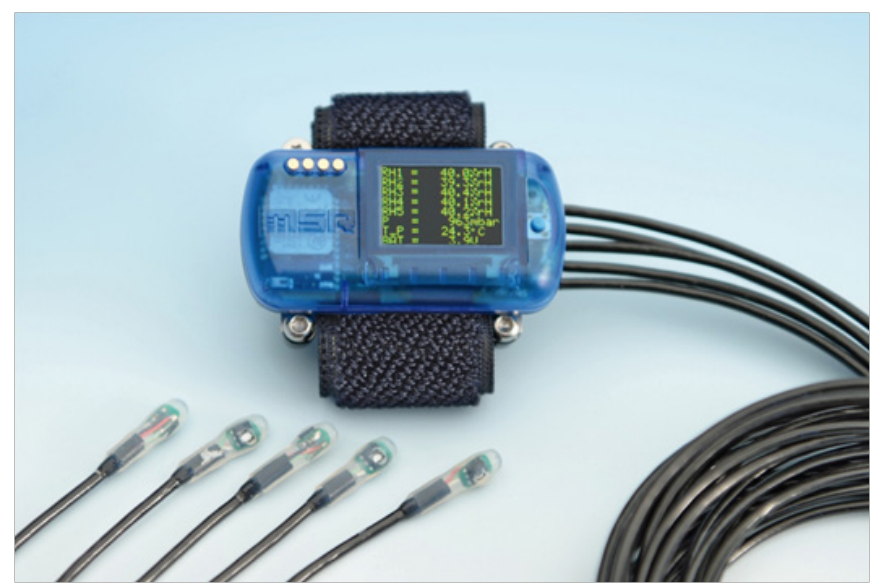

Figure 1 MSR147WD wireless data logger with plug-in temperature and humidity sensors.

\section{Conclusion}

Due to its compact format, modular design, high-capacity memory of one million measured values and very low power requirements, the MSR147WD wireless data loggers are suitable as an efficient solution for measuring the physiological properties of textiles.

For further information on the MSR147WD, please visit:

http://www.msr.ch/en/product/special_data_logger/msr-147wdwireless-data-logger-measure-physiological-properties-textiles/ 


\section{About MSR electronics GmbH}

The core competency of MSR Electronics GmbH is the development and production of miniature universal data loggers for a wide range of applications. Among MSR Electronics GmbH's customers are wellknown companies such as Alstom, BMW, Carl Zeiss, Fein, Festo, Nestlé, Novartis, Oerlikon Balzers, Orbital Sciences Corp., Osram, Robert Bosch, Siemens, W.L. Gore \& Associates, V-Zug and many more.

\section{Acknowledgements}

None

\section{Conflict of interest}

Authors declare there is no conflict of interest in publishing the article. 